\title{
First case of 18F-FACBC PET/CT-guided salvage radiotherapy for local relapse after radical prostatectomy with negative 11C-Choline PET/CT and multiparametric MRI: New imaging techniques may improve patient selection
}

\author{
Eugenio Brunocilla ${ }^{1}$, Riccardo Schiavina ${ }^{1}$, Cristina Nanni ${ }^{2}$, Marco Borghesi ${ }^{1}$, Matteo Cevenini ${ }^{1}$, \\ Enrico Molinaroli ${ }^{1}$, Valerio Vagnoni ${ }^{1}$, Paolo Castellucci ${ }^{2}$, Francesco Ceci ${ }^{2}$, Stefano Fanti ${ }^{2}$, \\ Caterina Gaudiano ${ }^{3}$, Rita Golfieri ${ }^{3}$, Giuseppe Martorana ${ }^{1}$ \\ ${ }^{1}$ Department of Urology, University of Bologna, S. Orsola-Malpighi Hospital, Bologna, Italy; \\ 2 Department of Nuclear Medicine, University of Bologna, S. Orsola-Malpighi Hospital, Bologna, Italy; \\ ${ }^{3}$ Department of Radiology, S. Orsola-Malpighi Hospital, Bologna, Italy.
}

\begin{abstract}
Summary We present the first case of salvage radiotherapy based on the results of 18F-FACBC PET/CT performed for a PSA relapse after radical prostatectomy. The patients underwent $11 C$ Choline PET/CT and multiparametric MRI that were negative while 18F-FACBC PET/CT visualized a suspected local relapse confirmed by transrectal ultrasound-guided biopsy. No distant relapse was detected. Thus the patient was submitted to salvage radiotherapy in the prostatic fossa. After 20 months of follow-up, the PSA was undetectable and 18F-FACBC PET/CT was negative. Salvage radiotherapy after surgery, provided that it is administered at the earliest evidence of the biochemical relapse, may improve cancer control and favourably influence the course of disease as well as the adjuvant approach. New imaging techniques may increase the

efficacy of the salvage radiotherapy thus helping in the selection of the patients. Preliminary clinical reports showed an improvement in the detection rate of $20-40 \%$ of 18F-FACBC in comparison with 11C-Choline for the detection of disease relapse after radical prostatecomy, rendering the $18 \mathrm{~F}-\mathrm{FACBC}$ the potential radiotracer of the future for prostate cancer.
\end{abstract}

KEY WORDS: Prostate cancer; PET/CT; 11C-Choline; 18FFACBC; Salvage radiotherapy; Biochemical relapse; Local relapse.

Submitted 12 September 2013; Accepted 30 June 2014

\section{INTRODUCTION}

About $30 \%$ of all patients undergoing radical prostatectomy (RP) or radiation therapy for prostate cancer ( $\mathrm{PCa}$ ) will develop local or distant recurrences within 10 years from initial therapy, and a third of patients will receive secondline treatment within five years $(1,2)$. Generally the biochemical relapse precedes the disease relapse by many years and disease relapse with undetectable PSA is exceptionally rare (3). Conventional imaging techniques are not adequate in localizing the site of recurrence in the early phase of the PSA relapse. Nowadays, Positron Emission Tomography/Computerized Tomography (PET/CT) with 11Carbonium or 18-Fluoro-Choline may identify the site of recurrence earlier, with better accuracy than conventional imaging and in a single step (4). However, Choline PET/CT has showed limited accuracy in the detection of local recurrence in patients with low level of PSA. Multiparametric magnetic resonance showed encouraging results to detect local recurrence in patients with low PSA and with small disease relapse (5). In recent years the investigational synthetic L-leucine analogue (antilamino-3-18F-fluorocyclobutane-1-carboxylic acid, in brief 18 F-FACBC) has been proposed as a possible alternative radiopharmaceutical to detect PCa relapse (6). From a clinical point of view the performance of $18 \mathrm{~F}$ FACBC for the evaluation of PCa relapse showed first very promising results (7). In the setting of the salvage treatments for disease relapse of PCa, many observational studies of salvage radiotherapy (RT) have shown complete responses in a substantial proportion of patients in the early phase of the PSA relapse with comparable results as adjuvant radiotherapy (6). We present the first case to our knowledge of salvage radiotherapy based on the results of $18 \mathrm{~F}-\mathrm{FACBC}$ PET/CT performed for a PSA relapse after radical prostatectomy.

\section{Case report}

57 years-old men was scheduled for radical treatment for clinical T2 prostate cancer with biopsy Gleason score of $4+3$ and preoperative PSA of $12.0 \mathrm{ng} / \mathrm{ml}$. Preoperative evaluation with bone scan and 11C-Choline PET/CT showed the absence of distant or lymphatic metastases. The patients underwent radical prostatectomy plus extended pelvic lymph-node dissection including inter- 
nal, external, obturator lymph-nodes and common lymph-nodes up to the cross of the ureters. The final histology after surgery showed a pT3a PCa with Gleason score $=4+4$ and no lymph-node metastases (LNMs) out 21 lymph-nodes retrieved. After 24 months from surgery, PSA increased up to $1.1 \mathrm{ng} / \mathrm{ml}$ (PSA doubling time of 10 months). Bone scan was negative. He underwent endorectal multiparametric MRI, 18F-FACBC PET/CT and 11CCholine PET/CT within one week in the setting of a comparative study protocol approved by the ethical committee of our Hospital (9). 11C-Choline PET/CT and MRI were negative (Figures 1, 2) while 18F-FACBC showed a positive uptake within prostatic fossa (Figure 3).

Transectal ultrasound-guided biopsy confirmed the presence of prostate cancer relapse (Gleason score $=4+4$ ) and intensity modulated radiotherapy (64.8 Gy) plus androgen deprivation therapy for 6 months were administrated. After 20 months after radiotherapy PSA was undetectable with no androgen deprivation therapy. No treatment complications were recorded.

Figures are posted in Supplementary materials on www.aiua.it

\section{Discussion}

Choline-PET/CT is nowadays the most important imaging technique in the assessment of PCa relapse and can detect the site of disease recurrence even with very low PSA level. The most important limitation is the limited spatial resolution of PET/CT scanner with little detection rate for small local relapse. Furthermore, when CholinePET/CT identifies a suspected lesion, almost twice as many metastases are present and the detection rate of Choline-PET/CT is still suboptimal (4). One important reason for this low sensitivity is the slow proliferation of PCa cells reflecting a slow membrane metabolism and resulting in a small amount of Choline uptake.

For this reason, some metastatic deposit even greater than $10 \mathrm{~mm}$ may be completely negative at PET/CT scan. Multiparametric MRI has demonstrated higher global accuracy than Choline-PET/CT for local relapse detection but small lesion can be missed (5).

The functional activity of the new radiotracer 18FFACBC for PET/CT is related to two different amino acid transporters (ASC and LAT1) which appears to be upregulated in prostate cancer progression to metastatic disease $(6,7)$.

Preliminary clinical reports with $18 \mathrm{~F}-\mathrm{FACBC}$ showed an improvement in the detection rate of $20-40 \%$ in comparison with 11C-Choline, rendering the 18F-FACBC the potential radiotracer of the future (7). Although data from randomized trials are lacking, substantial evidence from retrospective, observational studies shows that salvage RT is effective at controlling local recurrence and reduces the risk of distant metastasis and disease specific mortality $(1,8)$. New diagnostic tools such as $18 \mathrm{~F}-$ FACBC PET/CT may improve the potential of this approach by increasing the assessment and the selection of the patients.

The ability of 18F-FACBC PET/CT of visualizing the site of recurrence in the early phase of prostate cancer relapse may improve the tailoring of the treatment planning and may enhance the response to salvage treatments. The present clinical report and fist clinical studies encourage going on in the research of new imaging techniques in the detection of prostate cancer recurrence.

\section{REFERENCES}

1. Stephenson AJ, Bolla M, Briganti A, et al. Postoperative radiation therapy for pathologically advanced prostate cancer after radical prostatectomy. Eur Urol. 2012; 61:443-51.

2. Brunocilla E, Pultrone C, Pernetti R, et al. Preservation of the smooth muscular internal (vesical) sphincter and of the proximal urethra during retropubic radical prostatectomy: Description of the technique. Int J Urol. 2012; 19:783-5.

3. Pepe P, Fraggetta F, Tornabene F, et al Solitary lung metastasis after radical prostatectomy in presence of undetectable PSA. Arch Ital Urol Androl. 2012;84:208-10.

4. Farsad M, Schiavina R, Franceschelli A, et al. Positron-emission tomography in imaging and staging prostate cancer. Cancer Biomarker. 2008; 4:277-84.

5. Panebianco V, Sciarra A, Lisi D, et al. Prostate cancer: 1HMRSDCEMR at $3 T$ versus [(18)F]choline PET/CT in the detection of local prostate cancer recurrence in men with biochemical progression after radical retropubic prostatectomy (RRP). Eur J Radiol. 2012; 81:700-8.

6. Schuster DM, Savir-Baruch D, Nieh P, et al. Detection of recurrent prostate carcinoma with Anti-3-18F-Fluorocyclobutane-1Carboxylic Acid PET/CT and 111In-Capromab Pendetide SPECT/CT. Radiology 2011; 259:852-861.

7. Nanni C, Schiavina R, Boschi S, et al. Comparison of 18F-FACBC and $11 C$-Choline PET/CT in patients with radically treated prostate cancer and biochemical relapse: preliminary results. Eur J Nucl Med Mol Imaging 2013, Apr 17.

8. Tramacere F, Gianicolo EA, Pignatelli A, Portaluri M. Analysis of survival in radical and postoperative radiotherapy for prostate cancer. Arch Ital Urol Androl 2011; 83:188-94.

\author{
Correspondence \\ Eugenio Brunocilla, MD \\ Riccardo Schiavina, MD (Corresponding Author) \\ rschiavina@yahoo.it \\ Marco Borghesi, MD \\ Matteo Cevenini, MD \\ Enrico Molinaroli, MD \\ Valerio Vagnoni, $M D$ \\ Giuseppe Martorana, MD \\ Department of Urology of University of Bologna, S. Orsola-Malpighi \\ Hospital, Via Palagi 9, 40134, Bologna, Italy \\ Cristina Nanni, MD \\ Paolo Castellucci, MD \\ Francesco Ceci, MD \\ Stefano Fanti, MD \\ Department of Nuclear Medicine of University of Bologna, \\ S. Orsola-Malpighi Hospital, Bologna, Italy \\ Caterina Gaudiano, MD \\ Rita Golfieri, MD \\ Department of Radiology, S. Orsola-Malpighi Hospital, Bologna, Italy
}

